\title{
INVESTIGACIONES
}

\section{Evaluación de la escritura de estudiantes sordos bilingües*}

\author{
Writing Assessment of Deaf Bilingual Students \\ Avaliação da escritura de estudantes bilíngües surdos \\ Valeria Herrera Fernández, ${ }^{a}$ Daniela Chacón Macchiavello, ${ }^{b}$ \\ Felipe Saavedra Coronado \\ ${ }^{a}$ Centro de Investigación Avanzada en Educación, Universidad de Chile \\ Correo electrónico: valherrera@u.uchile.cl \\ bEscuela Básica Especial para Sordos No 1712 "Santiago Apóstol”. Municipalidad de Santiago \\ Correo electrónico: danielachaconm@outlook.com \\ 'Universidad Metropolitana de Ciencias de la Educación \\ Correo electrónico: felipesaavedra@ outlook.com
}

\begin{abstract}
RESUMEN
La perspectiva de Derechos de las Personas con Discapacidad concibe la Educación de Sordos en el marco de un Modelo Educativo Intercultural Bilingüe (EIB), donde la Lengua de Señas Chilena (LSCh) es la lengua natural y primera lengua (L1) de las personas sordas y el español la lengua de contacto con la comunidad oyente y segunda lengua (L2). Este cambio paradigmático se articula en este estudio en concepciones que dan sentido y sistematizan la práctica pedagógica. Se propone una rúbrica para evaluar 57 producciones escritas realizadas por 43 estudiantes sordos de $1^{\circ}$ a $4^{\circ}$ año básico, con el objetivo de monitorear la enseñanza del español como L2. Se evalúan siete criterios en cuatro niveles de desempeño. Las conclusiones muestran que los textos producidos por estudiantes sordos bilingües presentan características similares a las de los producidos por aprendices de segundas lenguas.
\end{abstract}

Palabras clave: evaluación, segunda lengua, sordera.

\section{ABSTRACT}

Legal perspective regarding people with disabilities conceives the education of deaf people in the Intercultural Bilingual Educational Model (EIB), where the Chilean Language of Signs (LSCh) is the natural language and the first language (L1) and the Spanish is the language of contact with the community and second language (L2). This perspective is expressed in this study in conceptions that give sense and systematize the pedagogic practice. It proposes a rubric to evaluate 57 written productions developed by 43 deaf students in 1st to 4 th grades of primary school, with the aim being to monitor the instruction of Spanish as L2. Seven criteria are evaluated in four levels of performance. The conclusions show that the texts produced by deaf bilingual students present similar characteristics to those produced by second languages learners.

Key words: assessment, second language, deafness.

Este estudio se realizó en el marco del Proyecto Fondecyt Regular N 1130182 Comprensión lectora en estudiantes sordos de $1^{\circ} a 4^{\circ}$ año básico. Estrategias visuales, cognitivas y metacognitivas y prácticas efectivas de enseñanza. 


\section{INTRODUCCIÓN}

Las personas sordas usuarias de LSCh poseen una lengua y cultura propias y conforman una comunidad que ha enfrentado diversas vivencias en relación a su cultura y educación. El Congreso de Milán (1880) instaura la lengua oral como medio exclusivo y excluyente de comunicación para las personas sordas a nivel mundial, negando las lenguas de señas. El Congreso de Hamburgo (1990) revierte esta situación y proclama el derecho de las personas sordas de ser educadas en su lengua natural y ser provistas de ambientes pedagógicos acordes con el procesamiento visual (Skliar, Massone y Veinberg, 1995). En las propuestas de EIB, la LSCh permite la comunicación, el acceso a la información, la socialización y el desarrollo del pensamiento. Mientras que el aprendizaje del español como L2 es un puente comunicativo entre personas sordas y oyentes. Sin embargo, la mayoría de los estudiantes sordos presentan dificultades al leer y escribir, estas dificultades se relacionan, entre otras causas, con los métodos de enseñanza utilizados. Al respecto, los estudiantes sordos poseen capacidades diferentes a las de los estudiantes oyentes y la adquisición del español por parte de cada grupo es diferente (Padden y Ramsey, 2000). En cuanto a la escritura de los estudiantes sordos, Lissi et al. (2003) señalan que presentan mayor retraso que en la lectura y no alcanzan el nivel lector esperado según los cursos en que se encuentran. Concluyen que la evaluación es compleja debido a que los resultados se comparan con los niños oyentes. Por ello, es necesario caracterizar las producciones escritas desde la perspectiva de aprendices de L2 utilizando instrumentos específicos. En este contexto, el objetivo de la investigación es analizar las producciones escritas de estudiantes sordos de $1^{\circ}$ a $4^{\circ}$ año básico, a partir de un instrumento de evaluación para la producción de textos y proponer algunas orientaciones pedagógicas desde una perspectiva de EIB.

\section{ENSEÑANZA DE LA LECTURA Y LA ESCRITURA EN LA EDUCACIÓN DE SORDOS}

A lo largo de la historia de la educación de sordos en el mundo, han surgido distintas conceptualizaciones y paradigmas en torno a las personas sordas. Durante siglos la discapacidad se definió desde la patología y el déficit buscando normalizar y equiparar la condición de este grupo a la de la mayoría, a través del desarrollo de distintas estrategias de comunicación oral, lectura labial y habla destinadas a la "normalización". El Congreso de Milán del año 1880 adhiere a los métodos del oralismo, cambiando los objetivos pedagógicos por objetivos terapéuticos (Herrera, 2010). El oralismo tradicional fue la única opción educativa hasta entrada la década del sesenta del siglo pasado, cuando surge la filosofía de la Comunicación Total basada en el uso de todos los medios posibles para comunicarse con los sordos. Alrededor de 1990 surge el bimodalismo, que contempla la utilización simultánea de habla y señas manteniendo la estructura sintáctica de la lengua oral. Hacia fines de 1990 comienzan las primeras experiencias en Chile de bilingüismo en la educación de sordos. Los estudios sobre la lingüística de las distintas lenguas de señas del mundo, junto con las concepciones de discapacidad emanadas del paradigma socioantropológico, permiten reconocer que las personas sordas pertenecen a una comunidad lingüística minoritaria, con características particulares, plenas capacidades de desarrollo y un sistema lingüístico propio (Pérez, 2002). William Stokoe, en 1960, define la ASL 
(American Sign Language) como un código de comunicación viso-gestual utilizado por las personas sordas, que al igual que cualquier lengua hablada, reúne todas las características morfológicas, gramaticales y sintácticas de una lengua. Es el medio para la construcción de mundo y el contacto temprano con personas sordas y oyentes. La utilización temprana de lengua de señas impacta positivamente en el desarrollo emocional, social y académico de las personas sordas (Padden y Ramsey, 2000).

Por otra parte, los estudios de Humphries y Padden (2005) sostienen que las personas sordas poseen una cultura propia y particular, tienen su propia lengua, son personas eminentemente visuales, se comunican con sus manos y cuerpo, conforman una comunidad en torno a la lengua de señas y comparten valores, ideas, creencias, hábitos y tradiciones que se transmiten en su lengua. Por ello, la relación con la comunidad de sordos es fundamental (de la Paz y Salamanca, 2009). El concepto de comunidad está ligado al de identidad sorda, la construcción de esta es un proceso que se desarrolla al momento de integrarse a las asociaciones y compartir las normas de convivencia y elementos simbólicos de la comunidad que determinan que la persona se identifique con sus pares sordos. En la perspectiva del paradigma socio-antropológico, surge como respuesta a las necesidades educativas, comunicativas y sociales de los estudiantes sordos el Modelo Bilingüe Bicultural (Fish y Morford, 2012). A partir de los estudios que avalan el estatus de las lenguas de señas, estas cobran valor en la educación de los sordos, al ser la lengua natural de este grupo y con la que se asegura la comunicación y el desarrollo cognitivo y social (Humphries y Padden, 2005). Junto con ello, la lengua oral de la comunidad oyente en la que se insertan los estudiantes adquiere importancia al ser el canal de comunicación con la sociedad y un medio de adquisición y transmisión de conocimiento (Robles, 2012; Ruiz, 2011).

El bilingüismo en la educación de los sordos sugiere un continuo que se extiende desde la competencia mínima hasta la perfección en las habilidades lingüísticas en las dos lenguas (Robles Gómez, 2012). Para adquirir una segunda lengua es primordial tener una lengua consolidada, ya que esto apoya el desarrollo fluido de una segunda lengua y se da una relación de equilibrio entre ambas (Cummins, 2002; Fish y Morford, 2012). Cummins (2002) explica esta relación en la Teoría de Interdependencia Lingüística (TIL) declarando que en la medida que la enseñanza de L1 sea eficaz para promover el dominio de L1, se producirá la transferencia de este dominio a L2. Los estudiantes sordos que poseen y utilizan la LSCh como L1, desarrollan la competencia comunicativa y gramatical a través de esta y es la LSCh la que se establece como lengua de instrucción e interacción mediante la que los estudiantes sordos aprenden la L2 (Herrera, 2009; Herrera, Puente y Alvarado, 2014). En este proceso es común que existan errores, que son el resultado de las estrategias de procesamiento del lenguaje que emplean los estudiantes sordos al aprender L2. Al comparar aprendices de segunda lengua sordos y oyentes Svartholm (2008 Cit. en Lissi et al., 2010) comprobó que ambos grupos obtenían resultados similares al simplificar, sobre generalizar, errar en la utilización de preposiciones e inflexiones verbales, etc.

Desde el Modelo de EIB el aprendizaje de L1 es la base para el aprendizaje de L2 y permite la interrelación de las comunidades de sordos y oyentes. En palabras de Salamanca y de la Paz (2009: 19) "la educación intercultural bilingüe es una propuesta ideológica de encuentro y de complementariedad entre culturas". Al respecto, los objetivos principales de la EIB se relacionan con proporcionar una identidad, las escuelas IB incluyen ambas culturas y lenguas, a través de la participación de oyentes y sordos que cumplen con el rol de modelos lingüísticos y culturales. La EIB busca asegurar el contacto con la LSCh para su 
adquisición temprana y transmisión de los contenidos curriculares de cada nivel educativo. Este modelo requiere profesores que compartan esta visión educativa, sean competentes en ambas lenguas para interactuar con los estudiantes (Easterbrook y Beas-Alvares, 2013), conozcan la comunidad sorda y promuevan sus formas culturales (Robles, 2012).

Las necesidades de aprendizaje de la escritura de los estudiantes sordos son particulares y no son comparables con las de los oyentes. Las dificultades que presentan son multifactoriales (Zambrano, 2002). En primer lugar, existe una relación directa entre el nivel de lengua de señas y las habilidades para comprender y producir lenguaje escrito. En segundo lugar, la falta de métodos de enseñanza del español como segunda lengua para estudiantes sordos (Alonso, 2006). Y en tercer lugar, la implementación de prácticas pedagógicas de enseñanza y metodologías de aprendizaje que los profesores puedan implementar. A esto se agrega el hecho de que los docentes no tienen claras las concepciones de lectura, escritura y alfabetización (Alonso, 2006; Page at al., 2009), lo que impide tener un modelo sistemático de enseñanza en la sala de clases.

Respecto de los modelos de lectura requeridos, Herrera (2009) sugiere considerar el modelo de lectura propuesto por Chamberlain, Morford y Mayberry (2000) fundado en la Teoría de Interdependencia Lingüística (Cummins, 2002) y la Visión Simple de la Lectura (VSL) formulada por Hoover y Grough (1990 Cit. en Herrera, 2009), que considera a la lectura como un proceso basado en la comprensión lingüística y en la decodificación. Chamberlain, Morford y Mayberry (2000) indican que este modelo de lectura, permite explicar con mayor pertinencia el desarrollo de la lectura en los estudiantes sordos, ya que la comprensión lingüística se torna fundamental en la comprensión lectora. Finalmente, lo que separa la comprensión lingüística de la comprensión lectora es la decodificación, deducir una representación desde el input impreso para acceder al léxico mental y, de este modo, recuperar la información semántica de la palabra (Herrera, 2009). Por su parte, Alegría y Domínguez (2009) plantean que se requiere de conocimientos específicos y no específicos para llegar a la comprensión lectora. Los conocimientos no específicos son saberes indispensables para comprender la frase escrita, pero no son específicos de la lectura, es el sentido de la lectura una vez que se ha decodificado. Por otra parte, los conocimientos específicos son necesarios para identificar palabras y la conexión de una serie de letras que al decodificar conforman un significado.

Por su parte, la escritura es una expresión y estructuración del pensamiento, una representación cognitiva, funcional y contextual y un instrumento de comunicación (Gutiérrez, 2004). Es fundamental para alcanzar otros conocimientos, para acceder a la información del medio y constituye un sistema comunicativo en sí, ya que tiene una finalidad comunicativa concreta (Cummins, 2002). Es una actividad social compleja que requiere la activación de conocimientos generales y lingüísticos relacionados con la fonética, ortografía, léxico, morfosintaxis, textualidad y pragmática. A su vez, implica la capacidad de gestionar y aplicar estos conocimientos, reflexionar acerca de la realidad, de sus percepciones, sus experiencias y permite producir estructuras cognitivas cada vez más complejas y abstractas (Gutiérrez, 2004).

Para Gutiérrez (2004) el texto se compone de macro, micro y superestructura. La macroestructura o dimensión discursiva del texto incluye aquellos elementos estructurales narrativos, los nexos de cohesión, es la representación lógica, cognitiva, semántica del contenido global del texto. La microestructura o dimensión formal del texto, considera las estructuras sintácticas, sus variantes y disfunciones. Son los recursos lingüísticos, referidos 
al nivel oracional o superficial que representan el significado del texto y que son necesarios para la continuidad del discurso. En tanto la superestructura se refiere al esquema textual común de todo texto, que establece el contenido semántico en función de los diferentes tipos textuales. Además, explica que para que un texto se conforme es imprescindible que exista cohesión y coherencia en su producción. La cohesión es sintáctica y léxica, es el enlace entre los elementos en el nivel formal o superficial, expresa la organización discursiva y la coherencia es pragmática y semántica, es la relación lógica entre los diferentes conceptos e ideas de un texto, en el nivel del significado, de la estructura profunda, debe atender a los factores del contexto material y social.

Al evaluar el trabajo realizado por los sordos en el ámbito de la escritura, así como en la lectura, se corroboran dificultades. Las personas sordas, en general, no son competentes en estas tareas. En su mayoría egresan analfabetos o con bajo conocimiento de la lengua escrita así como de la pragmática, la sintaxis y los elementos socioculturales de esta (Lissi et al., 2003, 2010; Massone, Buscaglia y Bogado, 2005, 2010). La base de estas dificultades no se encuentra en el déficit auditivo, sino que tiene relación con los escasos recursos comunicativos, lingüísticos, experienciales, contextuales y sociales que tienen los estudiantes sordos dada la ausencia o retraso en la aparición de un sistema de comunicación (Gutiérrez, 2012), lo que hace que encuentren muchas barreras para adquirir la L2 e interiorizar las convenciones de la lectura y la escritura (Pérez, 2002). Por otro lado, los procedimientos utilizados para enseñar la lectura y escritura, son los mismos que se utilizan para la enseñanza de los niños oyentes y han sido desarrollados mayoritariamente desde un enfoque fonológico. Por lo tanto, se convierte en una tarea muy difícil para los niños sordos, ya que sus experiencias comunicativas no son auditivas sino visuales (Easterbrook y Beas-Alvarez, 2013; Gutiérrez, 2004; Herrera, Puente y Alvarado, 2014).

Consecuentemente, es muy necesaria una propuesta educativa que considere un enfoque específico para el aprendizaje de L2 en estudiantes sordos (Herrera et al., 2014). Al respecto, Russel y Lapenda (2012) desarrollaron un programa de trabajo para el español como L2, dando apoyo a estudiantes sordos e inmigrantes en Argentina. Concluyen que los estudiantes sordos incluidos en este programa se desempeñaban de forma similar a la de los estudiantes de español como L2 y que ambos grupos, sordos y oyentes, no alcanzaban los resultados obtenidos por los estudiantes oyentes argentinos. Por su parte, Morales y Valles (2002) estudiaron la gramática de las producciones escritas de estudiantes sordos y encontraron errores morfosintácticos, de organización del texto, de coherencia y de cohesión del texto. Explican que estos errores se hallan también en las producciones de estudiantes oyentes que aún no aprenden todas las reglas de la lengua escrita o que están aprendiendo una L2. En la misma línea, Massone et al. (2005) analizaron las producciones escritas realizadas por estudiantes sordos fuera del contexto escolar, a través de correos enviados entre sordos y entre sordos y oyentes. Observaron una interferencia lingüística constante entre español y lengua de señas y que los sordos tratan de apropiarse de la L2 con estrategias propias de un extranjero aprendiendo otra lengua.

Albertini y Schley (2003) consideran que los estudiantes sordos pueden reconstruir el sistema del lenguaje escrito y que gran parte de este proceso es similar al que realizan los estudiantes oyentes y que solo hace falta el acceso a la comunicación desde temprana edad y la exposición a la lengua escrita en el medio ambiente. Señalan que los textos de los sordos han sido caracterizados por tener oraciones cortas y simples, errores sintácticos que se evidencian en ausencia o mal uso de nexos, conjunciones y preposiciones a nivel 
discursivo, vocabulario restringido y repetitivo. En el nivel sintagmático-pragmático no aplican las reglas de concordancia para género y número en sustantivos ni terminaciones verbales.

Por otra parte, los profesionales que evalúan los escritos de los estudiantes sordos deben considerar las dificultades que presentan en la tarea de escribir y centrar la evaluación en el significado y no en los errores propios del desarrollo que aparecen en el texto (Schmitz y Keenan, 2005). Considerando la evaluación como un proceso cuyo objetivo es retroalimentar el aprendizaje, es posible identificar el nivel en el que se encuentran los estudiantes respecto de un aprendizaje, para posteriormente adaptar la enseñanza a sus requerimientos y obtener un proceso constructivo (Condemarín y Medina, 2000). Contreras, González y Urías (2009) plantean utilizar la rúbrica para evaluar la escritura, esta es una escala en la que se describen uno o varios tipos y niveles de habilidad en una ejecución determinada. Presenta una forma cualitativa de representar los escritos de los estudiantes, evaluándolos y pudiendo compararlos a partir de una escala con un rango que contiene desde la respuesta excelente hasta la inapropiada.

Sotomayor et al. (2014) elaboraron una rúbrica para evaluar y otorgar una mayor retroalimentación a las instituciones educativas. Los criterios de análisis utilizados fueron coherencia, cohesión, estructura, adecuación a la situación comunicativa, puntuación y evaluación global del texto. Al evaluar textos escritos en L1 por estudiantes oyentes de $4^{\circ}$ año básico, detectaron que, en su mayoría, responden adecuadamente a la situación comunicativa. En relación a la coherencia dentro del texto pudieron identificar diferencias significativas de desempeño dependiendo del tipo de texto solicitado, limitado uso de conectores, alto conocimiento en estructuración de textos dependiendo de la tipología y, tras hacer una evaluación global, ubicaron las producciones en un nivel regular. El uso de este tipo de instrumento permite un análisis más exhaustivo del desempeño de los estudiantes por área y la retroalimentación del trabajo en las salas de clases desde la evaluación formativa.

\section{METODOLOGÍA}

La investigación presenta una perspectiva cualitativa descriptiva logrando una representación detallada de las producciones escritas de estudiantes sordos de escuelas bilingües. Se analizan 57 escritos en base a la rúbrica elaborada, que permitió caracterizar las producciones de manera individual y obtener una evaluación cualitativa para entender los casos en su totalidad (Stake, 2005). El estudio se realizó en dos escuelas bilingües para sordos ubicadas en la ciudad de Santiago. Participan 43 estudiantes sordos entre 7 y 13 años que cursan $1^{\circ}$ a $4^{\circ}$ año básico durante el año 2014. Antes de aplicar las pruebas, se solicitaron consentimientos informados a los padres y apoderados de cada uno de los niños.

El análisis se realizó en base a una rúbrica elaborada para este estudio. La rúbrica fue sometida a evaluación de contenido por juicio experto de parte de siete egresados de Pedagogía en Lenguaje y comunicación, Educación diferencial y Educación básica de la Universidad Metropolitana de Ciencias de la Educación. La rúbrica evalúa varios niveles de habilidad, donde se representa cualitativamente la actividad de acuerdo a los criterios específicos. La rúbrica orienta la medición de cada uno de los criterios, usando un rango para ordenar las respuestas. Los criterios utilizados en la rúbrica (Sotomayor et al., 2014) son: 
(1) Adecuación Comunicativa, implica que los estudiantes son capaces de ajustarse al tema y al propósito requerido. (2) Coherencia, referida al sentido global de la producción, la relación entre ideas y la coherencia temáticas. (3) Cohesión, referida al nivel sintáctico de las producciones, conexión entre las ideas y uso de conectores. (4) Estructura Textual, que implica ajustarse al tipo de texto que se desea escribir, incluyendo los elementos propios de cada tipo de texto: carta, noticia, etc. (5) Gramática, que implica concordancia entre la frase nominal y frase verbal evidenciada en las desinencias verbales y concordancias de género y número. (6) Ortografía, referida a escribir palabras según las normas de la lengua española. (7) Puntuación, referida al uso correcto de mayúscula y signos de puntuación. Cada uno de estos criterios contiene cuatro niveles que describen desde el desempeño mínimo hasta el máximo esperado, de manera de guiar la asignación de puntaje al evaluador (Tabla 1). Así, se logra dar un resultado cuantitativo que permite una descripción cualitativa detallada de las características de las producciones tanto a nivel individual y como grupal.

Tabla 1. Puntajes y niveles de desempeño para evaluar las producciones escritas

\begin{tabular}{cl}
\hline Puntaje & \multicolumn{1}{c}{ Descripción } \\
\hline 0 & No es posible evaluar debido a la poca extensión o incumplimiento del criterio. \\
1 & El criterio no se cumple o se logra con dificultad. \\
2 & El criterio se cumple a pesar de las dificultades o de la falta de aspectos a evaluar. \\
3 & El criterio se cumple totalmente. \\
\hline
\end{tabular}

\section{RÚBRICA PARA EVALUAR LAS PRODUCCIONES ESCRITAS DE ESTUDIANTES SORDOS DE $1^{\circ} \mathrm{A} 4^{\circ}$ AÑO BÁSICO}

\begin{tabular}{|c|c|c|c|}
\hline Criterio & Definición & Puntaje & Descripción \\
\hline \multirow{4}{*}{$\begin{array}{l}\text { Adecuación } \\
\text { a la situación } \\
\text { comunicativa }\end{array}$} & \multirow{4}{*}{$\begin{array}{l}\text { Se evidencia ajuste al } \\
\text { tema y propósito co- } \\
\text { municativo impuesto } \\
\text { por la escritura, a las } \\
\text { variables contextuales } \\
\text { que inciden en el re- } \\
\text { sultado final de la co- } \\
\text { municación: emisor, } \\
\text { receptor, intención } \\
\text { comunicativa. }\end{array}$} & 0 & $\begin{array}{l}\text { No se ajusta al tema ni al propósito, la informa- } \\
\text { ción no es clara o no hay suficiente escritura para } \\
\text { evaluarlo. }\end{array}$ \\
\hline & & 1 & $\begin{array}{l}\text { Se ajusta solo al tema o solo al propósito sin ma- } \\
\text { yor desarrollo, se presentan dificultades para } \\
\text { identificar la intención comunicativa. }\end{array}$ \\
\hline & & 2 & $\begin{array}{l}\text { Se ajusta al tema y al propósito parcialmente, no } \\
\text { integra emisor o receptor (cuando es necesario) y } \\
\text { se logra identificar la intención comunicativa. }\end{array}$ \\
\hline & & 3 & $\begin{array}{l}\text { Se ajusta al tema y al propósito comunicativo, } \\
\text { integrando emisor y receptor (cuando es necesa- } \\
\text { rio) y se logra identificar la intención comunica- } \\
\text { tiva de manera clara. }\end{array}$ \\
\hline
\end{tabular}




\begin{tabular}{|c|c|c|c|}
\hline \multirow{4}{*}{ Coherencia } & \multirow{4}{*}{$\begin{array}{l}\text { Se evidencia un senti- } \\
\text { do global completo en } \\
\text { las relaciones que en- } \\
\text { lazan las ideas del } \\
\text { texto y que crean sen- } \\
\text { tido en los lectores sin } \\
\text { digresiones temáticas. } \\
\text { Se identifican las } \\
\text { ideas principales a } \\
\text { través del texto. }\end{array}$} & 0 & $\begin{array}{l}\text { No se observa un sentido global, son recurrentes } \\
\text { las digresiones temáticas y las ideas principales } \\
\text { no se explicitan de manera clara o no hay sufi- } \\
\text { ciente escritura para evaluar. }\end{array}$ \\
\hline & & 1 & $\begin{array}{l}\text { Se observan dificultades para lograr un sentido } \\
\text { global del texto, no todas las ideas del texto se } \\
\text { entrelazan, presentando digresiones temáticas. } \\
\text { Las ideas principales se presentan con dificultad. }\end{array}$ \\
\hline & & 2 & $\begin{array}{l}\text { Se observa un sentido global del texto en las rela- } \\
\text { ciones de las ideas del texto, a pesar de las digre- } \\
\text { siones temáticas. Se identifican las ideas princi- } \\
\text { pales. }\end{array}$ \\
\hline & & 3 & $\begin{array}{l}\text { Se observa un sentido global en las relaciones de } \\
\text { las ideas del texto, sin digresiones temáticas. Se } \\
\text { identifican las ideas principales del texto. }\end{array}$ \\
\hline \multirow{4}{*}{ Cohesión } & \multirow{4}{*}{$\begin{array}{l}\text { Se evidencian relacio- } \\
\text { nes sintácticas entre } \\
\text { las partes, a partir del } \\
\text { uso de conectores. }\end{array}$} & 0 & $\begin{array}{l}\text { No se observan relaciones sintácticas entre las } \\
\text { palabras y las oraciones. Ausencia de conectores. } \\
\text { O el escrito no es suficiente para ser evaluado. }\end{array}$ \\
\hline & & 1 & $\begin{array}{l}\text { Se observan relaciones sintácticas con uso inade- } \\
\text { cuado de conectores entre palabras y oraciones. }\end{array}$ \\
\hline & & 2 & $\begin{array}{l}\text { Se observan relaciones sintácticas entre las pala- } \\
\text { bras y las oraciones a pesar de la falta de conec- } \\
\text { tores entre ellas. }\end{array}$ \\
\hline & & 3 & $\begin{array}{l}\text { Se observan relaciones sintácticas entre las pala- } \\
\text { bras y las oraciones a partir del uso adecuado de } \\
\text { conectores (conjunciones, preposiciones, conec- } \\
\text { tores). }\end{array}$ \\
\hline \multirow{4}{*}{$\begin{array}{l}\text { Estructura } \\
\text { Textual }\end{array}$} & \multirow{4}{*}{$\begin{array}{l}\text { Se evidencian las par- } \\
\text { tes de la estructura } \\
\text { convencional del tipo } \\
\text { de texto evaluado, que } \\
\text { organiza la informa- } \\
\text { ción de un modo par- } \\
\text { ticular para cumplir } \\
\text { su función comunica- } \\
\text { tiva. }\end{array}$} & 0 & $\begin{array}{l}\text { Ausencia de estructuras propias del tipo de texto } \\
\text { evaluado, no se presenta una estructura conven- } \\
\text { cional del tipo de texto evaluado o el escrito no es } \\
\text { suficiente para ser evaluado. }\end{array}$ \\
\hline & & 1 & $\begin{array}{l}\text { Ausencia de la mayoría de las partes o no están } \\
\text { bien definidas, se evidencian dificultades para } \\
\text { presentar la estructura convencional del tipo de } \\
\text { texto evaluado. }\end{array}$ \\
\hline & & 2 & $\begin{array}{l}\text { Están todas las partes y no están bien definidas, } \\
\text { pero se cumple la función comunicativa presen- } \\
\text { tando la estructura convencional del tipo de texto } \\
\text { evaluado. }\end{array}$ \\
\hline & & 3 & $\begin{array}{l}\text { Están todas las partes definidas, se cumple la fun- } \\
\text { ción comunicativa presentando la estructura con- } \\
\text { vencional del tipo de texto evaluado. }\end{array}$ \\
\hline
\end{tabular}




\begin{tabular}{|c|c|c|c|}
\hline \multirow{4}{*}{ Gramática } & \multirow{4}{*}{$\begin{array}{l}\text { Se evidencia concor- } \\
\text { dancia en los tiempos } \\
\text { verbales, género y nú- } \\
\text { mero entre el sujeto y } \\
\text { el verbo y en el uso de } \\
\text { artículos. }\end{array}$} & 0 & $\begin{array}{l}\text { No se presenta ningún tipo de concordancia o el } \\
\text { escrito no es suficiente para ser evaluado. }\end{array}$ \\
\hline & & 1 & $\begin{array}{l}\text { Se observan faltas de concordancia que impiden } \\
\text { la comprensión del texto. }\end{array}$ \\
\hline & & 2 & $\begin{array}{l}\text { Se observan faltas de concordancia, sin embargo } \\
\text { esto no afecta la comprensión. }\end{array}$ \\
\hline & & 3 & $\begin{array}{l}\text { Se observa concordancia en los tiempos verbales, } \\
\text { el género y el número, entre el sujeto y el predi- } \\
\text { cado cuando el texto lo requiera y en el uso de } \\
\text { artículos. }\end{array}$ \\
\hline \multirow{4}{*}{ Ortografía } & \multirow{4}{*}{$\begin{array}{l}\text { Se evidencia segui- } \\
\text { miento de normas del } \\
\text { español de acuerdo a } \\
\text { su nivel. }\end{array}$} & 0 & $\begin{array}{l}\text { No sigue las normas ortográficas acordes a su ni- } \\
\text { vel. }\end{array}$ \\
\hline & & 1 & $\begin{array}{l}\text { Hay faltas ortográficas que alteran la lectura o } \\
\text { comprensión del mensaje. }\end{array}$ \\
\hline & & 2 & $\begin{array}{l}\text { Hay faltas ortográficas, pero no alteran la com- } \\
\text { prensión del mensaje. }\end{array}$ \\
\hline & & 3 & $\begin{array}{l}\text { Todas las palabras siguen las normas ortográficas } \\
\text { acordes a su nivel. }\end{array}$ \\
\hline \multirow{4}{*}{ Puntuación } & \multirow{4}{*}{$\begin{array}{l}\text { Se evidencia uso de } \\
\text { puntuación externa } \\
\text { (mayúscula y punto } \\
\text { final) e interna (coma, } \\
\text { punto seguido, punto } \\
\text { aparte), uso de "y". }\end{array}$} & 0 & $\begin{array}{l}\text { Ausencia de elementos de puntuación necesarios } \\
\text { en el texto. }\end{array}$ \\
\hline & & 1 & $\begin{array}{l}\text { Comienza con mayúscula o acaba con punto final } \\
\text { o utiliza coma para enumerar. }\end{array}$ \\
\hline & & 2 & $\begin{array}{l}\text { Comienza con mayúscula y acaba con punto final } \\
\text { y utiliza coma para enumerar. }\end{array}$ \\
\hline & & 3 & $\begin{array}{l}\text { Comienza con mayúscula y acaba con punto fi- } \\
\text { nal; utiliza coma para enumerar y punto seguido } \\
\text { o aparte en el caso de desarrollar producciones } \\
\text { que lo ameriten. }\end{array}$ \\
\hline
\end{tabular}




\section{ANÁLISIS DE LOS RESULTADOS}

La unidad de análisis se compone de 57 (Tabla 2) producciones escritas realizadas por los estudiantes que participaron en el estudio, a partir de distintos estímulos entregados en cada nivel escolar. Los estímulos presentados fueron:

Primero básico: Observa la imagen y escribe una oración.

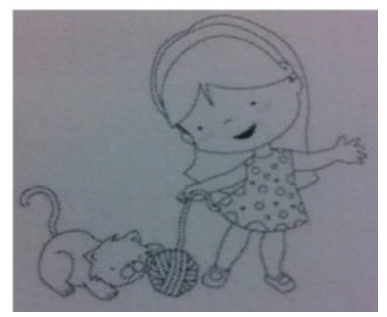

Segundo básico: Escribe una oración para cada lámina.
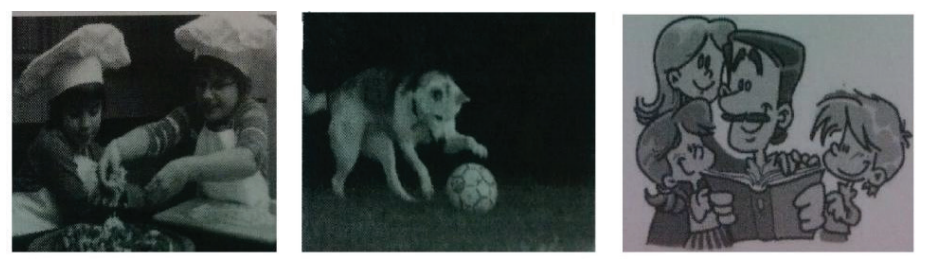

Tercero básico: Escribe una carta a un amigo contando lo que hiciste el fin de semana.

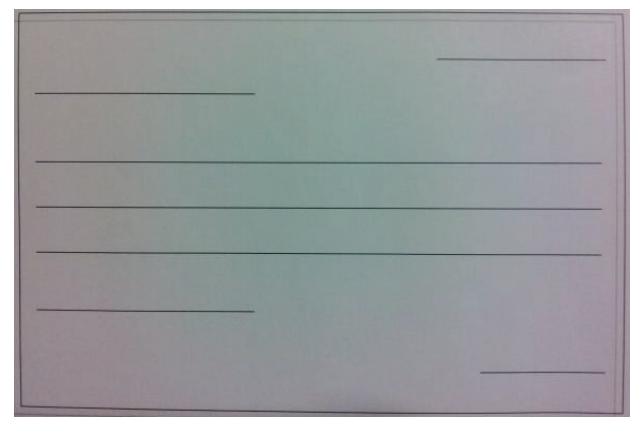


Cuarto básico: Escribe una noticia, contando ¿qué pasó?, ¿cuándo?, ¿dónde? y ¿cómo?

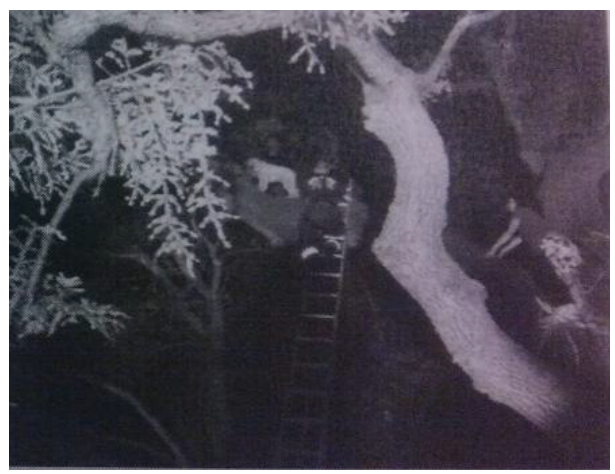

Tabla 2. Nivel escolar, producciones escritas, estudiantes y tipo de texto

\begin{tabular}{cccc}
\hline Nivel & $\mathrm{N}^{\circ}$ producciones & $\mathrm{N}^{\circ}$ estudiantes & Tipo de texto \\
\hline $1^{\circ}$ Básico & 14 & 14 & Oración \\
$2^{\circ}$ Básico & 23 & 9 & Oración \\
$3^{\circ}$ Básico & 12 & 12 & Carta \\
$4^{\circ}$ Básico & 8 & 8 & Noticia \\
\hline TOTAL & 57 & 43 & \\
\hline
\end{tabular}

A continuación se presenta un ejemplo de análisis con la evaluación realizada a partir de la rúbrica.

Primero básico: Observa la imagen y escribe una oración.
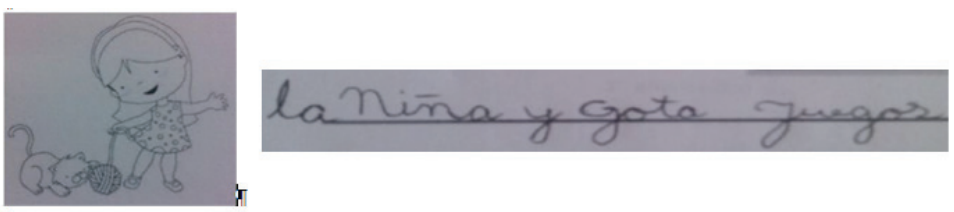


\begin{tabular}{|c|c|c|}
\hline \multicolumn{3}{|r|}{ Producción 1} \\
\hline Criterio & Puntaje & Descripción \\
\hline $\begin{array}{l}\text { Adecuación } \\
\text { a la situación } \\
\text { comunicativa }\end{array}$ & 3 & $\begin{array}{l}\text { Se ajusta al tema y al propósito comunicativo y se logra identificar la } \\
\text { intención comunicativa de manera clara. }\end{array}$ \\
\hline Coherencia & 3 & $\begin{array}{l}\text { Se observa un sentido global en las relaciones de las ideas del texto, } \\
\text { sin disgresiones temáticas. Se identifica la idea principal del texto. }\end{array}$ \\
\hline Cohesión & 3 & Se observa relación sintáctica a partir del uso de la conjunción " $y$ ". \\
\hline $\begin{array}{l}\text { Estructura } \\
\text { Textual }\end{array}$ & 2 & $\begin{array}{l}\text { Están todas las partes (frase nominal y frase verbal) requeridas para } \\
\text { el texto, aunque no están bien definidas, pero se cumple la función } \\
\text { comunicativa. }\end{array}$ \\
\hline Gramática & 2 & $\begin{array}{l}\text { Se observa falta de concordancia entre frase nominal y frase verbal } \\
\text { sin embargo esto no afecta la comprensión. }\end{array}$ \\
\hline Ortografía & 2 & Hay faltas ortográficas pero no alteran la comprensión del mensaje. \\
\hline Puntuación & 0 & Ausencia de elementos de puntuación necesarios en el texto. \\
\hline
\end{tabular}

\subsection{ANÁLISIS DE LAS PRODUCCIONES DE PRIMER AÑO BÁSICO}

A nivel global, 11 de las 14 producciones escritas por los estudiantes de primer año básico evaluados presentan un desempeño superior al 50\%. 7 de las 14 producciones logran entre el $61 \%$ y el $80 \%$ del desempeño esperado. En contraste, 3 de las 14 producciones evaluadas no superan el 33\% del desempeño esperado. En cuanto a los criterios evaluados, los mejor logrados son ortografía (2.5), coherencia (2.2) y adecuación a la situación comunicativa (2.0). Los criterios que presentan menor desempeño son puntuación (0.5), gramática (1.5), cohesión (1.5) y estructura textual (1.6). Estos resultados nos indican que, en general, las producciones escritas por estudiantes sordos bilingües se ajustan al tema y los propósitos requeridos, las palabras escritas se ajustan a las normas de la lengua española y presentan un nivel global coherente, relacionando ideas y temáticas. Por otra parte, se observan dificultades en el criterio puntuación, dificultades en cohesión, referida al nivel sintáctico, conexión en las ideas, uso de conectores, etc., gramática, en cuanto a concordancia entre la frase nominal y frase verbal y estructura textual.

En cuanto al criterio de adecuación a la situación comunicativa, las producciones escritas se ajustan al propósito comunicativo y al tema, logrando identificar la intención comunicativa de la oración solicitada. Las producciones realizadas son coherentes, se observa un sentido global del texto escrito y se identifica la idea principal de quien escribe. En más del $50 \%$ de las producciones escritas por los estudiantes de primer año básico hay ausencia de conectores, no obstante se puede encontrar un vínculo entre sus elementos y es posible comprender el sentido de las mismas. En los casos en que se presentan palabras 
con función gramatical, estas son de relación copulativa como: "y" y "que" y de relación preposicional como: "de". Se observa que algunas producciones integran claramente frase nominal y frase verbal incorporando sujeto y verbo, incluso complemento circunstancial de lugar. En algunas producciones se puede evidenciar la frase nominal de manera clara, pero la frase verbal es inexistente o está representada por una palabra que sugiere acción o un complemento.

En solo en una de las producciones se observa concordancia entre sujeto y verbo, sin embargo la falta de concordancia en el resto de producciones no afectó la comprensión. El uso de artículos, aunque mínimo, es adecuado y cumple con la concordancia de género y número. Los pocos errores ortográficos se relacionan con palabras que desconocen, aunque se mantiene el principio alfabético como principio básico de la escritura en español. Por ejemplo "cato" y "goto" en vez de gato, o "jagua" y "jesgos" en vez de jugar o juego. La mayoría de las producciones no presentan elementos de puntuación, sin embargo en algunos casos se observa el uso de mayúscula y en menor medida el uso de punto final. Como grupo las producciones escritas de los estudiantes de primero básico alcanzaron un porcentaje promedio total de desempeño de un $57.1 \%$ (Tabla 3 ).

Tabla 3. Evaluación de las producciones escritas de estudiantes de $1^{\circ}$ año básico

\begin{tabular}{|c|c|c|c|c|c|c|c|c|c|}
\hline 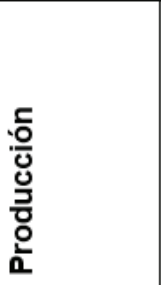 & 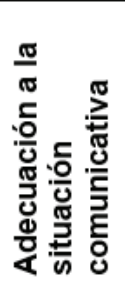 & 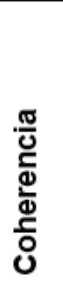 & 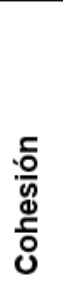 & 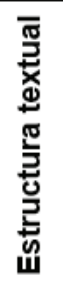 & 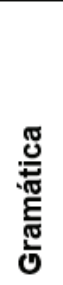 & 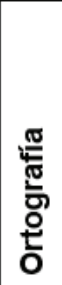 & 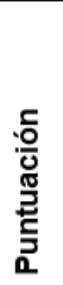 & 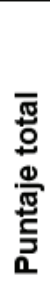 & 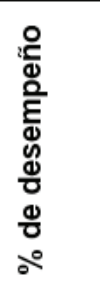 \\
\hline 1 & 3 & 3 & 3 & 2 & 2 & 2 & 0 & 15 & $71.4 \%$ \\
\hline 2 & 2 & 3 & 0 & 1 & 2 & 2 & 1 & 11 & $52.3 \%$ \\
\hline 3 & 1 & 1 & 0 & 1 & 0 & 3 & 1 & 7 & $33.3 \%$ \\
\hline 4 & 3 & 3 & 3 & 2 & 2 & 2 & 1 & 15 & $71.4 \%$ \\
\hline 5 & 1 & 1 & 0 & 1 & 0 & 3 & 1 & 7 & $33.3 \%$ \\
\hline 6 & 1 & 2 & 2 & 1 & 2 & 3 & 0 & 11 & $52.3 \%$ \\
\hline 7 & 3 & 3 & 2 & 2 & 2 & 1 & 0 & 13 & $61.9 \%$ \\
\hline 8 & 1 & 1 & 0 & 1 & 0 & 3 & 1 & 7 & $33.3 \%$ \\
\hline 9 & 3 & 3 & 3 & 1 & 2 & 3 & 1 & 16 & $76.1 \%$ \\
\hline 10 & 2 & 2 & 2 & 2 & 2 & 2 & 0 & 12 & $57.1 \%$ \\
\hline 11 & 3 & 3 & 3 & 3 & 2 & 2 & 0 & 16 & $76.1 \%$ \\
\hline 12 & 1 & 1 & 0 & 1 & 0 & 3 & 1 & 7 & $33.3 \%$ \\
\hline 13 & 2 & 3 & 2 & 2 & 2 & 3 & 0 & 14 & $66.6 \%$ \\
\hline 14 & 3 & 3 & 2 & 3 & 3 & 3 & 0 & 17 & $80.9 \%$ \\
\hline Promedio & 2.0 & 2.2 & 1.5 & 1.6 & 1.5 & 2.5 & 0.5 & 12 & $57.1 \%$ \\
\hline
\end{tabular}




\subsection{ANÁLISIS DE LAS PRODUCCIONES DE SEGUNDO AÑO BÁSICO}

A nivel global 18 de las 23 producciones escritas por los estudiantes de segundo año básico evaluados presentan un nivel de desempeño sobre el 50\%. 16 de las 23 producciones escritas superan el $60 \%$ del nivel de desempeño esperado. En contraste, el $21 \%$ de las producciones evaluadas no supera el $33 \%$ del desempeño esperado. En cuanto a los criterios evaluados los mejor logrados son ortografía (2.9), logrando casi el máximo puntaje como promedio, coherencia (2.3) y adecuación a la situación comunicativa (2.3). Los criterios que presentan menor desempeño son nuevamente puntuación (0.3), gramática (1.5), estructura textual (1.7) y cohesión (1.8). Estos resultados son muy coincidentes con los del nivel educativo precedente, mostrando que las producciones escritas por estudiantes sordos bilingües de segundo año básico mejoran sus desempeños en cuanto a que se ajustan al tema y los propósitos requeridos, las palabras escritas cumplen con las normas de la lengua española y presentan un nivel global coherente, relacionando ideas y temáticas, superior al logrado en el nivel anterior. Al igual que en las producciones de los estudiantes de primer año básico, se observan dificultades en puntuación, gramática, estructura textual y cohesión.

El análisis muestra la existencia de ajuste al tema y propósito comunicativo, por lo que se logra identificar la intención comunicativa de cada oración escrita. Se identifica la idea principal de quien escribe y a diferencia del nivel anterior, en general, las oraciones escritas presentan más de una palabra por lo que se puede evidenciar la relación entre los elementos. En más del $50 \%$ de las producciones hay ausencia de conectores, no obstante esto no implica que no existan relaciones sintácticas entre las palabras, ya que en la mayoría de las oraciones existen vínculos entre sus elementos y es posible comprender el sentido de las mismas. En los casos en que se presentan conectores, estos son las conjunciones "y", "que" y las preposiciones "de". En general, se observan oraciones con frase nominal y frase verbal, incorporando sujeto y verbo. En el resto de producciones se puede evidenciar la frase nominal sin acompañamiento de una frase verbal o con una palabra que sugiere acción o un complemento. En la mayoría de las producciones no hay concordancia entre sujeto y verbo, sin embargo la falta de concordancia entre los elementos no afectó la comprensión del mensaje. El uso de artículos aumenta en este nivel y su uso es adecuado, ya que cumple con la concordancia de género y número. No existen mayores errores ortográficos, al igual que en el nivel anterior se evidencian palabras que no corresponden al español. Las producciones no comienzan con mayúscula ni acaban con punto final. Tampoco hay comas para enumerar, necesarias para el tipo de oración más extensa. Las producciones de segundo básico alcanzaron un porcentaje promedio total de desempeño de un $61.9 \%$ (Tabla 4), logrando más de la mitad del nivel esperado en las producciones escritas. Seis producciones obtuvieron puntajes bajo el 50\%. El criterio mejor logrado fue ortografía (2.9) y el menos logrado fue puntuación (0.3). 
Tabla 4. Evaluación de las producciones escritas de estudiantes de segundo año básico

\begin{tabular}{|c|c|c|c|c|c|c|c|c|c|}
\hline $\begin{array}{l}\text { 응 } \\
\text { בूँ } \\
\text { 옴 }\end{array}$ & 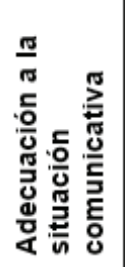 & 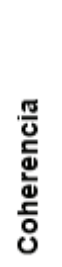 & 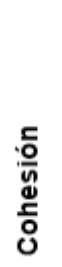 & 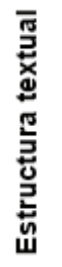 & 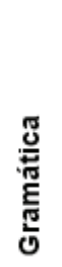 & 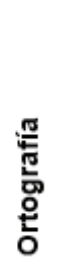 & 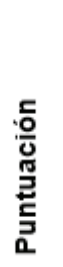 & 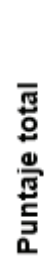 & 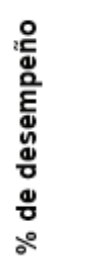 \\
\hline 15 & 1 & 1 & 0 & 1 & 0 & 3 & 0 & 6 & $28.5 \%$ \\
\hline 16 & 2 & 2 & 2 & 1 & 2 & 3 & 0 & 12 & $57.1 \%$ \\
\hline 17 & 3 & 2 & 2 & 1 & 2 & 3 & 1 & 14 & $66.6 \%$ \\
\hline 18 & 3 & 3 & 3 & 3 & 2 & 3 & 1 & 18 & $85.7 \%$ \\
\hline 19 & 3 & 3 & 3 & 1 & 3 & 3 & 1 & 17 & $80.9 \%$ \\
\hline 20 & 1 & 1 & 0 & 1 & 0 & 3 & 0 & 6 & $28.5 \%$ \\
\hline 21 & 2 & 3 & 2 & 2 & 2 & 3 & 0 & 14 & $66.6 \%$ \\
\hline 22 & 3 & 3 & 3 & 3 & 3 & 3 & 0 & 18 & $85.7 \%$ \\
\hline 23 & 3 & 3 & 3 & 3 & 2 & 3 & 0 & 17 & $80.9 \%$ \\
\hline 24 & 3 & 3 & 3 & 3 & 2 & 3 & 0 & 17 & $80.9 \%$ \\
\hline 25 & 3 & 3 & 3 & 3 & 2 & 3 & 0 & 17 & $80.9 \%$ \\
\hline 26 & 1 & 3 & 3 & 1 & 0 & 3 & 1 & 10 & $47.6 \%$ \\
\hline 27 & 2 & 2 & 2 & 1 & 2 & 3 & 1 & 14 & $66.6 \%$ \\
\hline 28 & 3 & 2 & 2 & 2 & 2 & 3 & 0 & 15 & $71.4 \%$ \\
\hline 29 & 3 & 2 & 2 & 2 & 2 & 3 & 0 & 15 & $71.4 \%$ \\
\hline 30 & 3 & 2 & 2 & 2 & 2 & 3 & 0 & 15 & $71.4 \%$ \\
\hline 31 & 3 & 2 & 2 & 2 & 2 & 3 & 0 & 15 & $71.4 \%$ \\
\hline 32 & 3 & 2 & 2 & 2 & 2 & 3 & 0 & 15 & $71.4 \%$ \\
\hline 33 & 1 & 0 & 0 & 1 & 0 & 3 & 1 & 7 & $33.3 \%$ \\
\hline 34 & 1 & 0 & 0 & 1 & 0 & 3 & 1 & 7 & $33.3 \%$ \\
\hline 35 & 1 & 0 & 0 & 1 & 0 & 3 & 0 & 6 & $28.5 \%$ \\
\hline 36 & 3 & 2 & 2 & 2 & 2 & 3 & 0 & 15 & $71.4 \%$ \\
\hline 37 & 3 & 1 & 1 & 1 & 2 & 2 & 0 & 11 & $52.3 \%$ \\
\hline Promedio & 2.3 & 2.3 & 1.8 & 1.7 & 1.5 & 2.9 & 0.3 & 13 & $61.9 \%$ \\
\hline
\end{tabular}

\subsection{ANÁLISIS DE LAS PRODUCCIONES DE TERCER AÑO BÁSICO}

A nivel global, 7 de las 12 producciones escritas por los estudiantes de tercer año básico evaluados presentan un nivel de desempeño superior al 50\% (Tabla 5). De ellas, 4 producciones escritas logran entre el $61 \%$ y el $90 \%$ del desempeño esperado. En contraste, 3 producciones no superan el $30 \%$ del desempeño esperado. En cuanto a los criterios evaluados, solo el criterio ortografía (2.5) supera el 50\% del puntaje de logro esperado. Los criterios coherencia (1.5), adecuación a la situación comunicativa (1.5) y estructura textual se presentan con un 50\% del puntaje esperado. Por su parte, los criterios de cohesión (1.2) y gramática no alcanzan el 50\% del puntaje esperado. Finalmente, el criterio puntuación (0.6), al igual que en los niveles precedentes, prácticamente no se expresa en las producciones escritas evaluadas. Estos resultados muestran un descenso en el desempeño global del grupo, comparado con los niveles escolares anteriores (primero y segundo básico). Las producciones escritas por los estudiantes de tercer año básico únicamente superan el $50 \%$ de logro en el criterio ortografía, presentando mayor dificultad en los criterios adecuación 
a la situación comunicativa, coherencia y estructura textual. Es decir, que los textos no se ajustan al tema y los propósitos requeridos, les falta sentido global, relación entre las ideas y coherencia temática y concordancia entre la frase nominal y frase verbal.

Debido a que, en su mayoría, las producciones evaluadas no se ajustan al tema o propósito, es difícil identificar la intención comunicativa de los estudiantes. Solo dos producciones logran este criterio, observando claramente una intención comunicativa e integran emisor y receptor, como en el caso planteado de escribir una carta identificando destinatario y remitente. Las producciones escritas logran escasamente un sentido global y expresión de ideas principales. Se observa variedad en las competencias sintácticas esperables en este criterio, los estudiantes que logran mejor desempeño en esta tarea se acercan a relaciones sintácticas que permiten comprender el texto ya sea con la presencia o ausencia de los conectores. En general las producciones de los estudiantes de tercer año básico presentan dificultades para desarrollar la estructura convencional de la carta aunque existen nociones básicas de la estructura del texto. Las variaciones que aparecen en las producciones de los estudiantes son notorias, se presentan solo frases nominales o solo frases verbales, en algunas producciones se logran construir las dos frases a pesar de las dificultades que se presentan en la conjugación verbal, cuando aparecen sin conjugación (en infinitivo). Las producciones inteligibles presentan ortografía acordes al nivel, en general hay ausencia de puntos seguidos y finales pero hay nociones del uso de mayúscula al comenzar las producciones. Algunos estudiantes usan signos de interrogación y exclamación cuando es necesario.

Las producciones escritas de los estudiantes de tercer año básico alcanzan un promedio total de desempeño de 50\%. Del total de estudiantes, cinco obtuvieron puntajes bajo el $50 \%$. El criterio mejor logrado fue ortografía (2.5) y el menos logrado puntuación (0.6).

Tabla 5. Evaluación de las producciones escritas de estudiantes de tercer año básico

\begin{tabular}{|c|c|c|c|c|c|c|c|c|c|}
\hline 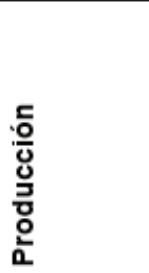 & 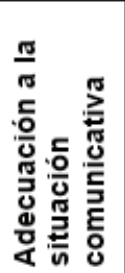 & 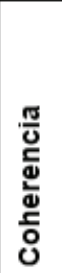 & 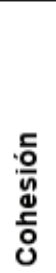 & 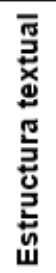 & 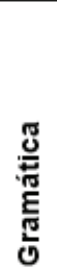 & 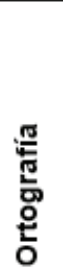 & 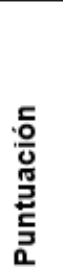 & 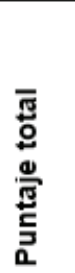 & 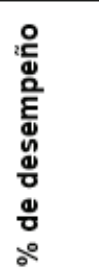 \\
\hline 38 & 1 & 1 & 1 & 1 & 1 & 3 & 0 & 8 & $38 \%$ \\
\hline 39 & 1 & 1 & 0 & 1 & 0 & 3 & 0 & 6 & $28.5 \%$ \\
\hline 40 & 1 & 1 & 0 & 2 & 1 & 3 & 0 & 8 & $38 \%$ \\
\hline 41 & 1 & 1 & 0 & 1 & 0 & 3 & 0 & 6 & $28.5 \%$ \\
\hline 42 & 0 & 0 & 0 & 0 & 0 & 0 & 0 & 0 & $0 \%$ \\
\hline 43 & 2 & 2 & 2 & 1 & 3 & 3 & 0 & 14 & $66.6 \%$ \\
\hline 44 & 2 & 2 & 2 & 1 & 1 & 3 & 1 & 13 & $61.9 \%$ \\
\hline 45 & 1 & 1 & 1 & 2 & 1 & 3 & 2 & 11 & $52.3 \%$ \\
\hline 46 & 2 & 2 & 2 & 2 & 2 & 2 & 0 & 12 & $57.1 \%$ \\
\hline 47 & 3 & 3 & 3 & 3 & 2 & 2 & 3 & 19 & $90.4 \%$ \\
\hline 48 & 2 & 2 & 2 & 1 & 2 & 3 & 0 & 11 & $52.3 \%$ \\
\hline 49 & 3 & 3 & 2 & 3 & 3 & 3 & 2 & 18 & $85.7 \%$ \\
\hline Promedio & 1.5 & 1.5 & 1.2 & 1.5 & 1.3 & 2.5 & 0.6 & 10.5 & $50 \%$ \\
\hline
\end{tabular}




\subsection{ANÁLISIS DE LAS PRODUCCIONES DE CUARTO AÑO BÁSICO}

A nivel global, 6 de las 8 producciones escritas por los estudiantes de cuarto año básico superan el $60 \%$ del nivel de desempeño esperado (Tabla 6). En contraste, dos producciones evaluadas no superan el $38 \%$ del desempeño esperado. En cuanto a los criterios evaluados, los mejores logrados son ortografía (2.6) y coherencia (2.0), seguidos por gramática (1.8), estructura textual (1.8) y coherencia (1.8); adecuación a la situación comunicativa (1.6) y puntuación (1.6). Los resultados muestran que las producciones escritas por estudiantes sordos bilingües se ajustan al tema y propósitos requeridos, las palabras escritas cumplen las normas de la lengua española, presentan un nivel global coherente, relacionando ideas y temáticas, concordancia entre la frase nominal y frase verbal, se ajustan al tipo de texto solicitado. Las producciones escritas presentan errores en cuanto a ajustarse al tema y al propósito y, al igual que todas las producciones evaluadas, ausencia de puntuación.

En general, las producciones se adecúan al tema, en el caso de la noticia, se cumple el propósito de informar. Se observa un sentido global del texto. Aunque en algunas producciones es difícil conocer la idea principal. En la mitad de las producciones se presenta relación entre las ideas del texto y las ideas principales se identifican sin dificultades. En la mayoría de las producciones se observan relaciones sintácticas entre las oraciones, a pesar de la falta de conectores entre ellas. Una producción (90\% de logro) presenta uso de preposiciones cuando el texto lo requiere. Las producciones cumplen la función comunicativa presentando la estructura convencional del tipo de texto, incluyendo uso de comillas en el título. Las producciones de los estudiantes de cuarto año básico presentan frase nominal y frase verbal, aunque no hay concordancia entre ellas. En las producciones en que sí hay concordancia, se observa la conjugación del verbo de acuerdo al sujeto. Las palabras cumplen las normas ortográficas de su nivel. Se observa uso de reglas de puntuación como mayúsculas y punto final. Las producciones de cuarto básico alcanzaron un porcentaje promedio total de desempeño de un $60.9 \%$, por lo que alcanzan más de la mitad de lo esperado en las producciones. Solo dos estudiantes obtuvieron puntajes bajo el 50\%, el criterio con menor desempeño fue el de puntuación (1.2) y el con mejor desempeño, el criterio de ortografía (2.6).

Tabla 6. Evaluación de las producciones escritas de estudiantes de cuarto año básico

\begin{tabular}{|c|c|c|c|c|c|c|c|c|c|}
\hline $\begin{array}{l}\text { 응 } \\
\text { 엉 } \\
\text { 흥 } \\
\text { 옹 }\end{array}$ & 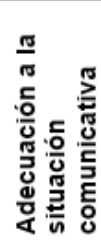 & 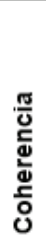 & $\begin{array}{l}\text { 음 } \\
\text { 등 }\end{array}$ & 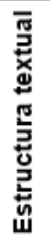 & 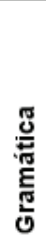 & 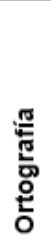 & 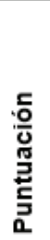 & 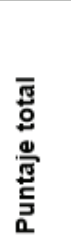 & 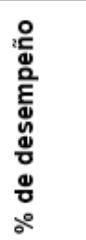 \\
\hline 50 & 3 & 2 & 2 & 2 & 2 & 3 & 1 & 15 & $71.4 \%$ \\
\hline 51 & 3 & 3 & 2 & 3 & 3 & 3 & 2 & 19 & $90.4 \%$ \\
\hline 52 & 0 & 0 & 0 & 1 & 0 & 1 & 0 & 2 & $9.5 \%$ \\
\hline 53 & 1 & 1 & 0 & 1 & 1 & 3 & 1 & 8 & $38 \%$ \\
\hline 54 & 2 & 2 & 2 & 2 & 2 & 3 & 2 & 15 & $71.4 \%$ \\
\hline 55 & 0 & 3 & 2 & 2 & 2 & 3 & 1 & 13 & $61.9 \%$ \\
\hline 56 & 3 & 3 & 3 & 2 & 3 & 3 & 1 & 18 & $85.7 \%$ \\
\hline 57 & 1 & 2 & 2 & 2 & 2 & 2 & 2 & 13 & $61.9 \%$ \\
\hline Promedio & 1.6 & 2.0 & 1.8 & 1.8 & 1.8 & 2.6 & 1.2 & 12.8 & $60.9 \%$ \\
\hline
\end{tabular}




\section{CONCLUSIONES}

La evaluación de la escritura de los estudiantes sordos se ha desarrollado a partir de instrumentos pensados para evaluar el desempeño de estudiantes oyentes. No obstante, el aprendizaje de la lengua escrita en el caso de estos estudiantes corresponde al aprendizaje de una segunda lengua y requiere de instrumentos que consideren este aspecto. La LSCh posee elementos sintácticos, morfosintácticos y gramaticales propios, que no tienen una transcripción literal al español y ello se debe considerar al evaluar la competencia en L2 de los estudiantes sordos. En este sentido, el uso de una rúbrica para evaluar la escritura resulta un aporte pedagógico concreto en la educación de sordos, permitiendo obtener una visión detallada de las habilidades de escritura en siete criterios específicos. La rúbrica entrega una guía para calificar las producciones escritas de los estudiantes en respuesta al mismo estímulo educativo, y a partir de ello realizar una descripción global de la producción (Contreras et al., 2009). Como señalan Schmitz y Keenan (2005), la evaluación debe enfocarse en el significado y no solo en los errores, que son parte del proceso de aprendizaje de una segunda lengua.

Los criterios de evaluación del instrumento se basan en rúbricas diseñadas por expertos en escritura, tomando los elementos necesarios para obtener un instrumento completo y que considere las dimensiones que se plasman en la producción de texto. Siguiendo a Gutiérrez (2004) y Sotomayor et al. (2014), estas dimensiones son coherencia, cohesión, gramática, sintaxis, ortografía, puntuación y estructura textual. Al evaluar las producciones escritas de los estudiantes sordos es necesario considerar la L1 como el primer sustento lingüístico para la construcción del pensamiento. En palabras de Fish y Morford (2012), a mayor competencia en L1, mayores son las posibilidades de consolidar una L2. La LSCh se plasma en la mayoría de las producciones y existe una interferencia lingüística permanente entre L1 y L2 que se expresa en errores de sintaxis, de concordancia y de gramática, al igual que sucede en el caso de otros aprendices de una segunda lengua como parte del procesamiento de las reglas de la L2 y no interfieren mayormente en la comprensión del mensaje (Albertini y Schley, 2005; Massone et al., 2005; Morales y Valles, 2002; Nivia y Valles, 2014; Russel y Lapenda, 2012).

En cuanto a los resultados en cada uno de los criterios evaluados, se puede señalar que si bien se presentaron debilidades en los resultados de cuarto básico, las competencias en ortografía, adecuación a la situación comunicativa y coherencia mejoran a medida que avanza el nivel educativo. Respecto a la puntuación, esta aparece como una debilidad que se presentó de manera constante en las evaluaciones de todas las producciones escritas en todos los niveles escolares. Al respecto, se sugiere trabajar estrategias que apunten sistemáticamente al uso de mayúsculas y punto seguido y final en contextos de aprendizaje significativo. Respecto a la ortografía, esta es una de las áreas mejor desarrolladas en todos los estudiantes sordos de $1^{\circ}$ a $4^{\circ}$ año básico, presentando un desempeño muy elevado. Este puede ser aprovechado a través del uso de estrategias morfográficas (morfología de palabras) para el aprendizaje y la ampliación de vocabulario en experiencias contextualizadas.

Respecto a la gramática, se evidencian debilidades en la flexión de verbos, concordancia entre el sujeto y la desinencia verbal, uso de artículos y concordancia entre género y número. Al respecto, se sugiere realizar actividades significativas de conjugación de verbos, entendiendo que la lengua natural de los estudiantes no presenta estos elementos, contrastar producciones en L1 y L2 para observar las diferencias e incluir en las producciones escritas 
etapas de revisión, borradores y edición en parejas o en forma grupal, sin perder de vista que se está realizando una enseñanza de L2 para estudiantes sordos.

En cuanto a cohesión, se observan pocas relaciones sintácticas, es decir un escaso uso de nexos como preposiciones y conjunciones inexistentes en la lengua de señas. Sin embargo, se pueden apreciar relaciones sintácticas y semánticas entre las palabras, en base a las últimas los docentes pueden reforzar este criterio trabajando de manera sistemática el uso de conectores, explicando a través del contraste la aplicación de reglas sintácticas de manera de trabajar en base a la competencia lingüística y luego en el nivel de la codificación, ambos necesarios en el aprendizaje de la segunda lengua (Herrera, 2009). En cuanto a la coherencia, las producciones muestran que los estudiantes construyen un sentido global del texto, a pesar de que no existe una mayor profundización en el escrito. Así se invita a los docentes a trabajar las ideas principales en la primera lengua para entender lo que el estudiante desea expresar, y luego trabajar con borradores de manera constructiva en conjunto. Respecto a la estructura textual, las principales debilidades se relacionan con la ausencia de elementos, que puede ser trabajada en base a la presentación de los diferentes tipos de textos y sus elementos, otorgando un contexto significativo para el estudiante: cartas a la familia, noticias en la escuela, recetas que se aplican en el aula, etc.

Finalmente, en cuanto a la adecuación a la situación comunicativa, las producciones presentan ajustes tanto al tema como al propósito comunicativo requerido por el estímulo. Se invita a los docentes a fomentar el uso de las producciones con temas y propósitos específicos, tanto en la lengua de señas como en la transcripción a la segunda lengua apoyándose de las tipologías textuales y se recomienda que la rúbrica u otro instrumento de evaluación sean conocidos por los estudiantes y sean utilizados a lo largo de todo el proceso educativo para obtener información de las fortalezas y las debilidades del estudiante en un sentido formativo. Por último, siempre es necesario que la producción de textos se fomente dentro del aula de clases, a partir de la tipología textual y dentro de un contexto significativo, es importante considerar algunas estrategias específicas para la producción de textos: grabar la producción en lengua de señas y luego trabajar en conjunto la transcripción a la segunda lengua, transparentar los criterios a evaluar, entregar estímulos adecuados y que no hagan que la tarea se torne tediosa, por lo tanto respetar los ritmos de los estudiantes.

\section{REFERENCIAS BIBLIOGRÁFICAS}

Albertini, J., \& Schley, S. (2003). Writing Characteristics, Instruction and Assessment. In Oxford Handbook of Deaf Studies, Language and Education (pp. 123-135). New York: Oxford University Press.

Alegría, J., \& Domínguez, A. (2009). Los alumnos sordos y la lengua escrita. Revista Latinoamericana de Educación Inclusiva, 3(1), 95-111.

Alonso, M. (2006). Métodos de enseñanza de lectura y escritura en personas sordas. Una mirada desde la práctica. Universidad Nacional de Cuyo: Argentina.

Chamberlain, Ch., Morford, J., \& Mayberry, R. (2000). Language Acquisition by Eye. Mahwah, N. J.: Lawrence Erlbaum Ass.

Condemarín, M., \& Medina, A. (2000). Evaluación de los aprendizajes, un medio para mejorar las competencias lingüísticas y comunicativas. Programa de Mejoramiento de la Calidad de las Escuelas Básicas de Sectores Pobres (P-900). Santiago: Ministerio de Educación de Chile. 
Contreras, L., González, M., \& Urías, E. (2009). Evaluación de la escritura mediante rúbrica en la educación primaria en México. Revista Interamericana de psicología, 43(3), 518-531.

Cummins, J. (2002). Lenguaje, poder y pedagogía. Madrid: Morata.

De la Paz, V., \& Salamanca, M. (2009). Elementos de la cultura sorda: Una base para el currículum intercultural. Revista de Estudios y Experiencias en Educación, 8(15), 31-49.

Easterbrooks, S., \& Beal-Alvarez, J. (2013). Literacy acquisition in students who are deaf and hard of hearing. Professional Perspectives on Deafness: Evidence and Applications. New York, NY: Oxford University Press.

Fish, S., \& Morford, J. (2012). NSF Science of Learning Center on Visual Language and Visual Learning. Research Brief $\mathrm{N}^{\circ} 7$ : The Benefits of Bilingualism. Recuperado desde: http://vl2. gallaudet.edu/files/5613/9216/6289/research-brief-7-the-benefits-of-bilingualism.pdf

Gutiérrez, R. (2004). Cómo escriben los alumnos sordos. Aljibe: Málaga.

Gutiérrez, R. (2012). Análisis del proceso de transcripción en la expresión escrita de alumnos sordos. Revista Complutense de Educación, 2, 331-346.

Herrera, V. (2009). En busca de un modelo educativo y de lectura coherente con las necesidades educativas especiales de los estudiantes sordos. Revista Rexe, 8(16), 11-24.

Herrera, V. (2010). Estudio de la población sorda en chile: evolución histórica y perspectivas lingüísticas, educativas y sociales. Revista Latinoamericana de Educación Inclusiva, 4(1), 211226.

Herrera, V., Puente, A., \& Alvarado, J.M. (2014). Visual learning strategies to promote literacy skills in prelingually deaf readers. Revista Mexicana de Psicología, 31(1), 1-10.

Humphries, T., \& Padden, C. (2005). Inside Deaf Culture. Cambridge: Harvard University Press.

Lissi, M. R., Grau, V., Raglianti, M., Salinas, M., \& Cabrera, I. (2003). Literacidad en escolares sordos chilenos: Evaluación y desafíos para la investigación y la educación. Revista Psykhe, 12(2), 37-57.

Lissi, M.R., Salinas, M., Acuña, X., Adamo, D., Cabrera, I., \& González, M. (2010). Using sign language to teach written language: an analysis of the strategies used by teachers of deaf children in a bilingual context. Educational studies in language and literature, 10(1), 57-69.

Massone, M.I., Buscaglia, V., \& Bogado, A. (2005). Los sordos aprenden a escribir sobre la marcha. Revista Lectura y vida, 26(4), 1-17.

Massone, M. I., Buscaglia, V., \& Bogado, A. (2010). La comunidad sorda: del trazo a la lengua escrita Revista Lectura y Vida, 1, 6-17.

Morales, A., \& Valles, B. (2002). Análisis de fenómenos gramaticales en la escritura de escolares sordos venezolanos. En Morales (Ed.), Educación para Sordos. Caracas: Fondo Editorial de la Universidad Pedagógica Experimental Libertador.

Nivia, D., \& Valles, B. (2014). Una aproximación a los fundamentos de la evaluación de la comprensión de la lectura en sordos: el caso venezolano. Revista Lenguaje, 42(2), 259-288.

Padden, C., \& Ramsey, C. (2000). American Sign Language and reading ability in deaf children. In Ch. Chamberlain, J. P. Morford y R. I. Mayberry (Eds.), Language Acquisition by Eye (pp. 165189). Mahwah, N. J.: Lawrence Erlbaum Ass.

Page, J., Lederberg, A., Easterbrooks, S., Malone, E., \& Mcdonald, C. (2009). Building the alphabetic principle in Young children who are deaf or hard of hearing. The volta review, 109(2), 87-119.

Pérez, Y. (2002). La producción de cuentos escritos por escolares sordos: una experiencia pedagógica con base en la lingüística textual. Investigación y Postgrado, 17(2), 11-52.

Robles, M. (2012). Tendencia educativa bilingüe y bicultural para la educación del sordo. Un nuevo camino hacia la inclusión. Unirevista, 1, 76-86.

Ruiz, E. (2011). Cohesión en el discurso narrativo escrito de estudiantes sordos (Tesis no publicada para maestría en lingüística). Universidad autónoma de Querétaro, México.

Russell, G., \& Lapenda, M. (2012). Un estudio comparativo sobre la enseñanza de la escritura a alumnos sordos. Revista signo y seña, 22, 63-85. 
Schmitz, K., \& Keenan, S. (2005). Evaluating deaf students' writing fairly: Meaning over mode. Rochester Institute of Technology (RIT) scholar works. Recuperado desde: http://scholarworks. rit.edu/cgi/viewcontent.cgi?article $=1628 \&$ context $=$ article

Skliar, C., Massone, M.I., \& Veinberg, S. (1995). El acceso de los niños sordos al Bilingüismo y al Biculturalismo. Revista Infancia y Aprendizaje, 69-70, 85-100.

Sotomayor, C., Gómez, G., Jéldrez, E., Bedwell, P., \& Domínguez, A.M. (2014). Calidad de la escritura en la educación básica. Documento de Trabajo $\mathrm{N}^{\circ}$ 13. Centro de Investigación Avanzada: Universidad de Chile.

Stake, R. (2005). Investigación con estudios de casos (3ra ed.). Madrid: Ediciones Morata.

Zambrano, L. (2002). El español escrito como segunda lengua para sordos ¿utopía o realidad? Revista Lingua Americana, VI(11), 65-76. 
